\title{
Microstructural Characterization of Aerospace Materials via Serial Sectioning using the Dual Beam FIB-SEM
}

\author{
Michael D. Uchic,* Michael Groeber** Robert Wheeler IV***, Robert Kerns***, Frank \\ Scheltens*** and Dennis M. Dimiduk* \\ * Air Force Research Laboratory, Materials \& Manufacturing Directorate, AFRL/MLLM, Wright- \\ Patterson AFB, OH 45433-7817, USA \\ ** The Ohio State University, Department of Materials Science and Engineering, 2041 College \\ Road, Columbus, OH 43210, USA \\ *** UES Inc., 4401 Dayton-Xenia Road, Dayton, OH 45432-1894, USA
}

This paper describes the application of a Dual Beam Focused Ion Beam-Scanning Electron Microscope to characterize the microstructure and crystallography of aerospace materials in three dimensions (3D). The Dual Beam microscope is capable of in-situ, high-fidelity serial sectioning, where the serial sectioning slice thickness can be less than 100 nanometers, and the interrogated volume can be on the order of $50 \times 50 \times 50$ micrometers in dimension. Another benefit is the potential availability of electron-beam characterization methods such as Energy Dispersive Spectroscopy (EDS) and Electron Backscatter Diffraction (EBSD), which can be integrated with the 3D-characterization experiment.

In order to take advantage of all of the analytical capabilities of DB 235, control scripts have been developed to automate the collection of serial sectioning data [1,2]. Automation of this application is essential, as it ensures that the serial-section slice thickness is consistent, and allows for examination of much larger volumes of material than if performed manually. Specifically, the software control scripts incorporate the use of built-in image recognition capabilities of the DB 235 to automatically move a sample to various spatial positions between serial sectioning events, and realign the sample prior to each serial-sectioning cut. At present, two fiducial marks are used to ensure that sample position is correct prior to cutting, imaging, or performing some other analysis, which allows the software to correct for both translational and rotational errors. The application of the image recognition methodology eliminates problems associated with stopping and re-starting an experiment, and also enables high-fidelity sectioning over the course of long-duration experiments (over multiple days).

With this control script, one can now perform fully-automated serial-sectioning experiments that acquire much more data types than just SEM images. For example, after cutting the sample can be moved and imaged using the ion beam (at a lower beam current than is used for cutting), to obtain an image exhibiting strong channeling contrast, as shown in Fig. 1a. One can also reposition the sample to acquire an EBSD or EDS maps, as shown in Fig. 1b. Of note, TSL Inc. has recently modified their acquisition software to allow for the collection of EBSD maps using a remote trigger, thus enabling the automated acquisition of EBSD maps during the serial sectioning experiment [3]. In the associated presentation, we will discuss the collection and post-processing of the serial sectioning data, segmentation routines for semi-automated identification of selected microconstituents, 3D reconstruction results, and preliminary analysis of selected microconstituents in $2 \mathrm{D}$ and $3 \mathrm{D}$.

[1] M. Groeber et al., Proc. Numiform 2004. 
[2] M.D. Uchic et.al, Microsc. Mircoanal. 10 (suppl. 2) 2004, 1136.

[3] The authors gratefully acknowledge financial support for this work from the Air Force Office of Scientific Research, under the direction of Dr. Craig Hartley, and the DARPA Accelerated Insertion of Materials Program. MG, FS, RK and RW further acknowledge support through the Materials \& Manufacturing Directorate through contract \#'s F33615-01-2-5225 and F33615-03-C-5206. The authors would also like to acknowledge Stuart Wright, Joe Ullmer, Paul Scutts, Mike Tiner, and Damian Dingley of TSL, Inc., for their assistance in incorporating the TSL OIM system into the serial sectioning scripting program.



Figure 1. Two ion images from a serial-sectioning experiment. The material is a polycrystalline Nibase superalloy, and the change in depth between the two images is $500 \mathrm{~nm}$.

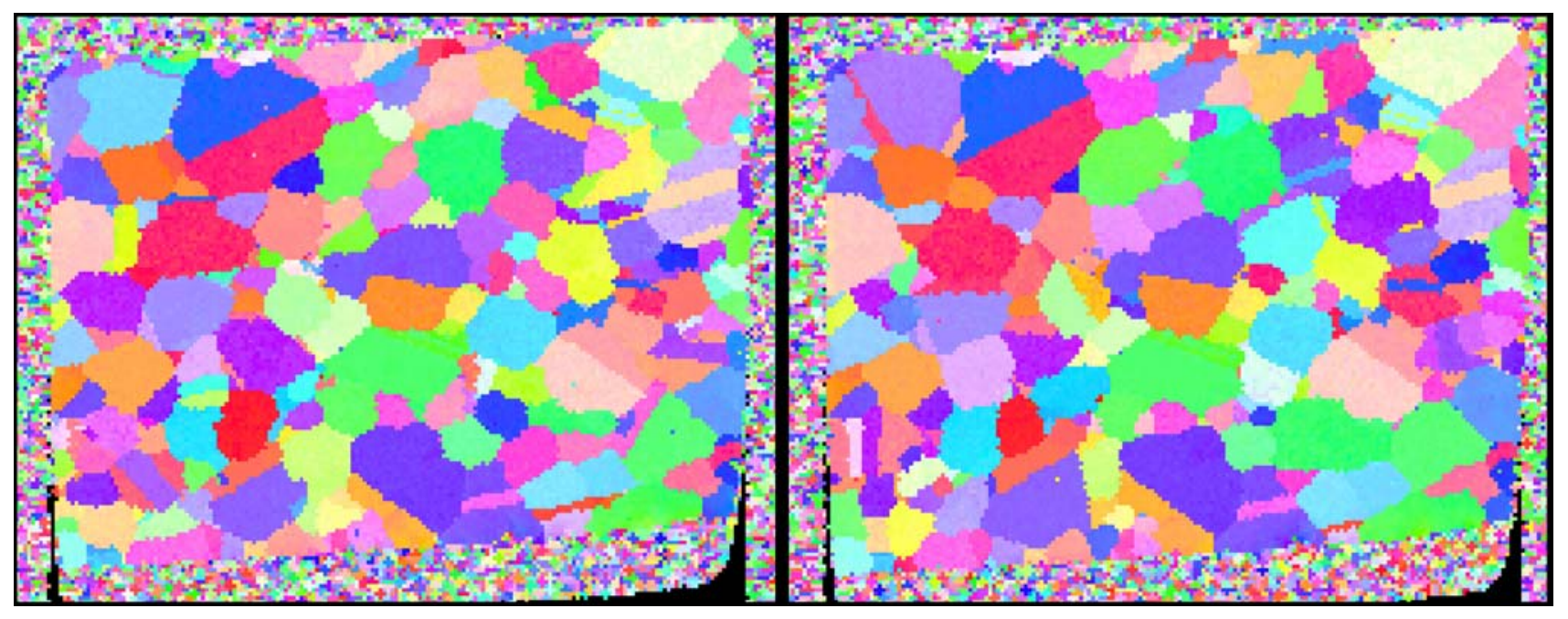

Figure 2. Two EBSD Inverse Pole Figure Maps that correspond to the images shown in Fig. 1. The data was obtained without user intervention during the serial sectioning experiment using the remote-trigger-capable TSL software. 